\title{
Covering Directed Graphs by In-trees
}

\author{
Naoyuki KAMIYAMA* ${ }^{*}$ Naoki $\mathrm{KATOH}^{\dagger}$
}

October 25, 2018

\begin{abstract}
Given a directed graph $D=(V, A)$ with a set of $d$ specified vertices $S=\left\{s_{1}, \ldots, s_{d}\right\} \subseteq V$ and a function $f: S \rightarrow \mathbb{Z}_{+}$where $\mathbb{Z}_{+}$denotes the set of non-negative integers, we consider the problem which asks whether there exist $\sum_{i=1}^{d} f\left(s_{i}\right)$ in-trees denoted by $T_{i, 1}, T_{i, 2}, \ldots, T_{i, f\left(s_{i}\right)}$ for every $i=1, \ldots, d$ such that $T_{i, 1}, \ldots, T_{i, f\left(s_{i}\right)}$ are rooted at $s_{i}$, each $T_{i, j}$ spans vertices from which $s_{i}$ is reachable and the union of all arc sets of $T_{i, j}$ for $i=1, \ldots, d$ and $j=1, \ldots, f\left(s_{i}\right)$ covers $A$. In this paper, we prove that such set of in-trees covering $A$ can be found by using an algorithm for the weighted matroid intersection problem in time bounded by a polynomial in $\sum_{i=1}^{d} f\left(s_{i}\right)$ and the size of $D$. Furthermore, for the case where $D$ is acyclic, we present another characterization of the existence of in-trees covering $A$, and then we prove that intrees covering $A$ can be computed more efficiently than the general case by finding maximum matchings in a series of bipartite graphs.
\end{abstract}

\section{Introduction}

The problem for covering a graph by subgraphs with specified properties (for example, trees or paths) is very important from practical and theoretical viewpoints and have been extensively studied. For example, Nagamochi and Okada [10] studied the problem for covering a set of vertices of a given undirected tree by subtrees, and Arkin et al. 1 studied the problem for covering a set of vertices or edges of a given undirected graph by subtrees or paths. These results were motivated by vehicle routing problems. Moreover, Even et al. 22 studied the covering problem motivated by nurse station location problems.

This paper studies the problem for covering a directed graph by rooted trees which is motivated by the following evacuation planning problem. Given a directed graph which models a city, vertices model intersections and buildings, and arcs model roads connecting these intersections and buildings. People exist not only at vertices but also along arcs. Suppose we have to give several evacuation instructions for evacuating all people to some safety place. In order to avoid disorderly confusion, it is desirable that one evacuation instruction gives a single evacuation path for each person and these paths do not cross each other. Thus, we want each evacuation instruction to become an in-tree rooted at some safety place. Moreover, the number of instructions for each safety place is bounded in proportion to a size of each safety place.

*Department of Architecture and Architectural Engineering, Kyoto University, Kyotodaigaku-Katsura, Nishikyo-ku, Kyoto, 615-8540, Japan. E-mail: is.kamiyama@archi.kyoto-u.ac.jp Supported by JSPS Research Fellowships for Young Scientists.

${ }^{\dagger}$ Supported by the project New Horizons in Computing, Grant-in-Aid for Scientific Research on Priority Areas, MEXT Japan. Department of Architecture and Architectural Engineering, Kyoto University, KyotodaigakuKatsura, Nishikyo-ku, Kyoto, 615-8540, Japan. E-mail: naoki@archi.kyoto-u.ac.jp 
The above evacuation planning problem is formulated as the following covering problem defined on a directed graph. We are given a directed graph $D=(V, A, S, f)$ which consists of a vertex set $V$, an arc set $A$, a set of $d$ specified vertices $S=\left\{s_{1}, \ldots, s_{d}\right\} \subseteq V$ and a function $f: S \rightarrow \mathbb{Z}_{+}$where $\mathbb{Z}_{+}$denotes the set of non-negative integers. In the above evacuation planning problem, $S$ corresponds to a set of safety places, and $f\left(s_{i}\right)$ represents the upper bound of the number of evacuation instructions for $s_{i} \in S$. For each $i=1, \ldots, d$, we define $V_{D}^{i} \subseteq V$ as the set of vertices in $V$ from which $s_{i}$ is reachable in $D$, and we define an in-tree rooted at $s_{i}$ which spans $V_{D}^{i}$ as a $\left(D, s_{i}\right)$-in-tree. We define a set $\mathcal{T}$ of $\sum_{i=1}^{d} f\left(s_{i}\right)$ subgraphs of $D$ as a $D$-canonical set of in-trees if $\mathcal{T}$ contains exactly $f\left(s_{i}\right)\left(D, s_{i}\right)$-in-trees for every $i=1, \ldots, d$. If every two distinct in-trees of a $D$-canonical set $\mathcal{T}$ of in-trees are arc-disjoint, we call $\mathcal{T}$ a $D$-canonical set of arc-disjoint in-trees. Furthermore, if the union of arc sets of all in-trees of a $D$-canonical set $\mathcal{T}$ of in-trees is equal to $A$, we say that $\mathcal{T}$ covers $A$.

Four in-trees illustrated in Figure 2 compose a $D$-canonical set $\mathcal{T}$ of in-trees which covers the arc set of a directed graph $D=(V, A, S, f)$ illustrated in Figure 1(a) where $S=\left\{s_{1}, s_{2}, s_{3}\right\}$, $f\left(s_{1}\right)=2, f\left(s_{2}\right)=1$ and $f\left(s_{3}\right)=1$. However, $\mathcal{T}$ is not a $D$-canonical set of arc-disjoint in-trees.

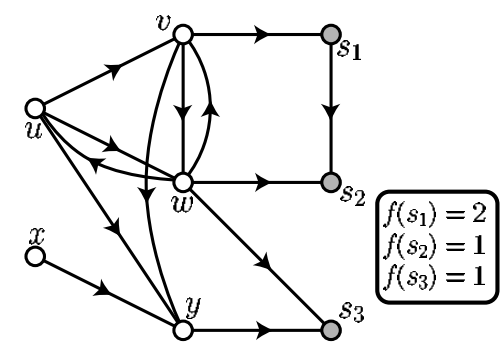

(a)

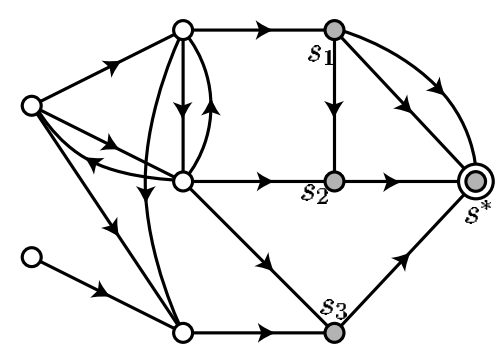

(b)

Figure 1: (a) Directed graph D. (b) Transformed graph $D^{*}$.

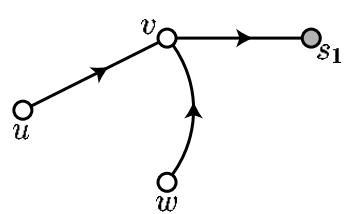

(a)

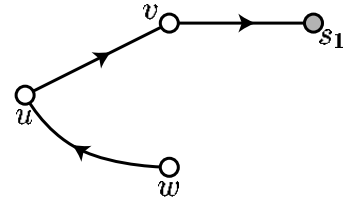

(b)

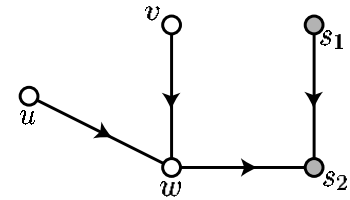

(c)

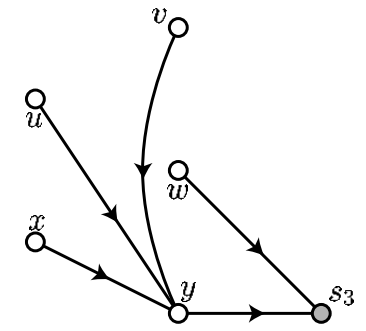

(d)

Figure 2: (a) $\left(D, s_{1}\right)$-in-tree. (b) $\left(D, s_{1}\right)$-in-tree. (c) $\left(D, s_{2}\right)$-in-tree. (d) $\left(D, s_{3}\right)$-in-tree.

We will study the problem for covering directed graphs by in-trees (in short CDGI), and we will present characterizations for a directed graph $D=(V, A, S, f)$ for which there exists a feasible solution of $\operatorname{CDGI}(D)$, and a polynomial time algorithm for $\operatorname{CDGI}(D)$.

\begin{aligned} & \hline Problem: $\operatorname{CDGI}(D) \\ &$\hline Input: a directed graph $D ; \\ &$ Output: a $D$-canonical set of in-trees which covers the arc set of $D$, if one exists. \\ & \hline\end{aligned}

A special class of the problem $\operatorname{CDGI}(D)$ in which $S$ consists of a single vertex was considered by Vidyasankar [13. He showed the necessary and sufficient condition in terms of linear inequalities 
that there exists a feasible solution of this problem (a weaker version was shown by Frank [4]). However, to the best of our knowledge, an algorithm for CDGI $(D)$ was not presented.

We will summerize our results as follows.

1. We first show that $\operatorname{CDGI}(D)$ can be viewed as some type of the connectivity augmentation problem. After this, we will prove that this connectivity augmentation problem can be solved by using an algorithm for the weighted matroid intersection problem in time bounded by a polynomial in $\sum_{i=1}^{d} f\left(s_{i}\right)$ and the size of $D$ (this generalizes the result by Frank [3]).

2. For the case where $D$ is acyclic, we show another characterization for $D$ that there exists a feasible solution of $\operatorname{CDGI}(D)$. Moreover, we prove that in this case $\operatorname{CDGI}(D)$ can be solved more efficiently than the general case by finding maximum matchings in a series of bipartite graphs instead of using an algorithm for the weighted matroid intersection problem.

\section{$1.1 \quad$ Outline}

The rest of this paper is organized as follows. Section 2 gives necessary definitions and fundamental results. In Section 3, we give an algorithm for the problem CDGI by using an algorithm for the weighted matroid intersection problem. In Section 4, we consider the acyclic case.

\section{Preliminaries}

Let $D=(V, A, S, f)$ be a connected directed graph which may have multiple arcs. Let $S=$ $\left\{s_{1}, \ldots, s_{d}\right\}$. Since we can always cover by $|A|\left(D, s_{i}\right)$-in-trees the arc set of the subgraph of $D$ induced by $V_{D}^{i}$, we consider the problem by using at most $|A|\left(D, s_{i}\right)$-in-trees. That is, without loss of generality, we assume that $f\left(s_{i}\right) \leq|A|$. For $B \subseteq A$, let $\partial^{-}(B)$ (resp. $\partial^{+}(B)$ ) be a set of tails (resp. heads) of arcs in $B$. For $e \in A$, we write $\partial^{-}(e)$ and $\partial^{+}(e)$ instead of $\partial^{-}(\{e\})$ and $\partial^{+}(\{e\})$, respectively. For $W \subseteq V$, we define $\delta_{D}(W)=\left\{e \in A: \partial^{-}(e) \in W, \partial^{+}(e) \notin W\right\}$. For $v \in V$, we write $\delta_{D}(v)$ instead of $\delta_{D}(\{v\})$. For two distinct vertices $u, v \in D$, we denote by $\lambda(u, v ; D)$ the local arc connectivity from $u$ to $v$ in $D$, i.e., $\lambda(u, v ; D)=\min \left\{\left|\delta_{D}(W)\right|: u \in W, v \notin\right.$ $W, W \subseteq V\}$. We call a subgraph $T$ of $D$ forest if $T$ has no cycle when we ignore the direction of $\operatorname{arcs}$ in $T$. If a forest $T$ is connected, we call $T$ tree. If every $\operatorname{arc}$ of an $\operatorname{arc}$ set $B$ is parallel to some arc in $A$, we say that $B$ is parallel to $A$. We denote a directed graph obtained by adding an $\operatorname{arc}$ set $B$ to $A$ by $D+B$, i.e., $D+B=(V, A \cup B, S, f)$. For $S^{\prime} \subseteq S$, let $f\left(S^{\prime}\right)=\sum_{s_{i} \in S^{\prime}} f\left(s_{i}\right)$. For $v \in V$, we denote by $R_{D}(v)$ a set of vertices in $S$ which are reachable from $v$ in $D$. For $W \subseteq V$, let $R_{D}(W)=\bigcup_{v \in W} R_{D}(v)$.

For an arc set $B$ which is parallel to $A$, we clearly have for every $v \in V$

$$
R_{D}(v)=R_{D+B}(v) .
$$

From (1), we have for every $i=1, \ldots, d$

$$
V_{D}^{i}=V_{D+B}^{i} .
$$

We define $D^{*}$ as a directed graph obtained from $D$ by adding a new vertex $s^{*}$ and connecting $s_{i}$ to $s^{*}$ with $f\left(s_{i}\right)$ parallel arcs for every $i=1, \ldots, d$ (see Figure 1). We denote by $A^{*}$ the arc set of $D^{*}$. From the definition of $D^{*}$,

$$
\left|A^{*}\right|=\sum_{v \in V}\left|\delta_{D^{*}}(v)\right|=|A|+f(S) .
$$


We say that $D$ is $(S, f)$-proper when $\left|\delta_{D^{*}}(v)\right| \leq f\left(R_{D}(v)\right)$ holds for every $v \in V$.

\subsection{Rooted arc-connectivity augmentation by reinforcing arcs}

Given a directed graph $D=(V, A, S, f)$, we call an arc set $B$ with $A \cap B=\emptyset$ which is parallel to $A$ a $D^{*}$-rooted connector if $\lambda\left(v, s^{*} ; D^{*}+B\right) \geq f\left(R_{D}(v)\right)$ holds for every $v \in V$. Notice that since a $D^{*}$-rooted connector $B$ is parallel to $A, B$ does not contain an arc which is parallel to an arc entering into $s^{*}$ in $D^{*}$. Then, the problem rooted arc-connectivity augmentation by reinforcing arcs (in short RAA-RA) is formally defined as follows.

\begin{aligned} \hline Problem: & RAA-RA $\left(D^{*}\right) \\$\hline Input: & $D^{*}$ of a directed graph $D ; \\$ Output: & a $D^{*}$-rooted connector $B$ whose size is minimum among all $D^{*}$-rooted \\ & connectors. \end{aligned}

Notice that the problem RAA-RA $\left(D^{*}\right)$ is not equivalent to the local arc-connectivity augmentation problem with minimum number of reinforcing arcs from $v \in V$ to $s_{i} \in R_{D}(v)$. For example, we consider $D^{*}$ illustrated in Figure 3 (a) of a directed graph $D=(V, A, S, f)$ where $S=\left\{s_{1}, s_{2}\right\}, f\left(s_{1}\right)=2$ and $f\left(s_{2}\right)=2$. The broken lines in Figure 3(b) represent a minimum $D^{*}$-rooted connector. For the problem that asks to increase the $v-s_{i}$ local arc-connectivity for every $v \in V$ and $s_{i} \in R_{D}(v)$ to $f\left(s_{i}\right)$ by adding minimum parallel arcs to $A$ (this problem is called the problem increasing arc-connectivity by reinforcing arcs in [7, in short $\operatorname{IARA}\left(D^{*}\right)$ ), an optimal solution is a set of broken lines in Figure 3(c). While it is known [7] that IARA $\left(D^{*}\right)$ is $\mathcal{N} \mathcal{P}$-hard, it is known [3] that RAA-RA $\left(D^{*}\right)$ in which $S$ consists of a single element can be solved in time bounded by a polynomial in $f(S)$ and the size of $D$ by using an algorithm for the weighted matroid intersection.

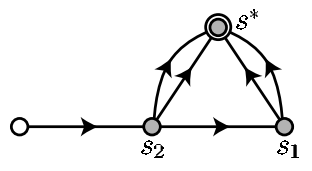

(a)

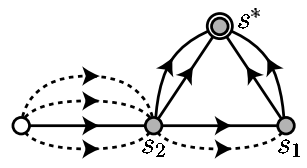

(b)

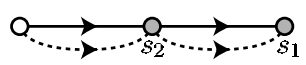

(c)

Figure 3: (a) Input. (b) Optimal solution for RAA-RA. (c) Optimal solution for IARA.

\subsection{Matroids on arc sets of directed graphs}

In this subsection, we define two matroids $\boldsymbol{M}\left(D^{*}\right)$ and $\boldsymbol{U}\left(D^{*}\right)$ on $A^{*}$ for a directed graph $D=(V, A, S, f)$, which will be used in the subsequent discussion. We denote by $\boldsymbol{M}=(E, \mathcal{I})$ a matroid on $E$ whose collection of independent sets is $\mathcal{I}$. Introductory treatment of a matroid is given in [11].

For $i=1, \ldots, d$ and $j=1, \ldots, f\left(s_{i}\right)$, we define $\boldsymbol{M}_{i, j}\left(D^{*}\right)=\left(A^{*}, \mathcal{I}_{i, j}\left(D^{*}\right)\right)$ where $I \subseteq A^{*}$ belongs to $\mathcal{I}_{i, j}\left(D^{*}\right)$ if and only if both of a tail and a head of every arc in $I$ are contained in $V_{D}^{i} \cup\left\{s^{*}\right\}$ and a directed graph $\left(V_{D}^{i} \cup\left\{s^{*}\right\}, I\right)$ is a forest. $\boldsymbol{M}_{i, j}\left(D^{*}\right)$ is clearly a matroid (i.e. graphic matroid). Moreover, we denote the union of $\boldsymbol{M}_{i, j}\left(D^{*}\right)$ for $i=1, \ldots, d$ and $j=1, \ldots, f\left(s_{i}\right)$ by $\boldsymbol{M}\left(D^{*}\right)=\left(A^{*}, \mathcal{I}\left(D^{*}\right)\right)$ in which $I \subseteq A^{*}$ belongs to $\mathcal{I}\left(D^{*}\right)$ if and only if $I$ can be partitioned into $\left\{I_{i, 1}, \ldots, I_{i, f\left(s_{i}\right)}: i=1, \ldots, d\right\}$ such that each $I_{i, j}$ belongs to $\mathcal{I}_{i, j}\left(D^{*}\right) . \boldsymbol{M}\left(D^{*}\right)$ is also a matroid (see Chapter 12.3 in [11. This matroid is also called matroid sum). When $I \in \mathcal{I}\left(D^{*}\right)$ can be partitioned into $\left\{I_{i, 1}, \ldots, I_{i, f\left(s_{i}\right)}: i=1, \ldots, d\right\}$ such that a directed graph $\left(V_{D}^{i} \cup\left\{s^{*}\right\}, I_{i, j}\right)$ is a tree for every $i=1, \ldots, d$ and $j=1, \ldots, f\left(s_{i}\right)$, we call $I$ a base of $\boldsymbol{M}\left(D^{*}\right)$. 
Next we define another matroid. We define $\boldsymbol{U}\left(D^{*}\right)=\left(A^{*}, \mathcal{J}\left(D^{*}\right)\right)$ where $I \subseteq A^{*}$ belongs to $\mathcal{J}\left(D^{*}\right)$ if and only if $I$ satisfies

$$
\left|\delta_{D^{*}}(v) \cap I\right| \leq \begin{cases}f\left(R_{D}(v)\right), & \text { if } v \in V \\ 0, & \text { if } v=s^{*}\end{cases}
$$

Since $\boldsymbol{U}\left(D^{*}\right)$ is a direct sum of uniform matroids, $\boldsymbol{U}\left(D^{*}\right)$ is also a matroid (see Exercise 7 of pp.16 and Example 1.2.7 in [11]). We call $I \in \mathcal{J}\left(D^{*}\right)$ a base of $\boldsymbol{U}(D)$ when (44) holds with equality.

For two matroids $\boldsymbol{M}\left(D^{*}\right)$ and $\boldsymbol{U}\left(D^{*}\right)$, we call an arc set $I \subseteq A^{*} D^{*}$-intersection when $I \in \mathcal{I}\left(D^{*}\right) \cap \mathcal{J}\left(D^{*}\right)$. If a $D^{*}$-intersection $I$ is a base of both $\boldsymbol{M}\left(D^{*}\right)$ and $\boldsymbol{U}\left(D^{*}\right)$, we call $I$ complete.

When we are given a weight function $w: A^{*} \rightarrow \mathbb{R}_{+}$where $\mathbb{R}_{+}$denotes the set of non-negative reals, we define the weight of $I \subseteq A^{*}$ (denoted by $w(I)$ ) by the sum of weights of all arcs $I$. The weighted matroid intersection problem (in short WMI) is then defined as follows [5].

\begin{aligned} & \hline Problem: $\operatorname{WMI}\left(D^{*}\right) \\ &$\hline Input: $D^{*}$ of a directed graph $D$ and a weight function $w: A^{*} \rightarrow \mathbb{R}_{+} ; \\ &$Output: a complete $D^{*}$-intersection $I$ whose weigh is minimum among all complete \\ &$D^{*}$-intersections, if one exists. \\ & \hline\end{aligned}

Lemma 2.1 We can solve $W M I\left(D^{*}\right)$ in $O\left(M\left|A^{*}\right|^{6}\right)$ time where $M=\sum_{v \in V} f\left(R_{D}(v)\right)$.

Proof. To prove the lemma, we use the following theorem concerning a matroid.

Theorem 2.2 ([9]) Given a matroid $M=(E, \mathcal{I})$ which is a union of $t(\leq|E|)$ matroids $\boldsymbol{M}_{1}=\left(E, \mathcal{I}_{1}\right), \ldots, \boldsymbol{M}_{t}=\left(E, \mathcal{I}_{t}\right)$, we can test if a given set belongs to $\mathcal{I}$ in $O\left(|E|^{3} \gamma\right)$ time where $\gamma$ is the time required to test if a given set belongs to $\mathcal{I}_{1}, \ldots, \mathcal{I}_{t}$.

Theorem 2.3 ([5]) Given two matroids $\boldsymbol{M}_{1}=\left(E, \mathcal{I}_{1}\right)$ and $\boldsymbol{M}_{2}=\left(E, \mathcal{I}_{2}\right)$ with a weight function $w: E \rightarrow \mathbb{R}_{+}$and a non-negative integer $k \in \mathbb{Z}_{+}$, we can find $I \in \mathcal{I}_{1} \cap \mathcal{I}_{2}$ with $|I|=k$ whose weight is minimum among all $I^{\prime} \in \mathcal{I}_{1} \cap \mathcal{I}_{2}$ with $\left|I^{\prime}\right|=k$ in $O\left(k|E|^{3}+k|E|^{2} \gamma\right)$ time if one exists where $\gamma$ is the time required to test if a given set belongs to both $\mathcal{I}_{1}$ and $\mathcal{I}_{2}$.

We consider the time required to test if a given set belongs to both $\mathcal{I}\left(D^{*}\right)$ and $\mathcal{J}\left(D^{*}\right)$. Since it is not difficult to see that we can test is a given set belongs to each $\mathcal{I}_{i, j}\left(D^{*}\right)$ in $O\left(\left|A^{*}\right|\right)$ time, we can test if a given set belongs to $\mathcal{I}\left(D^{*}\right)$ in $O\left(\left|A^{*}\right|^{4}\right)$ time from Theorem 2.2, For $\mathcal{J}\left(D^{*}\right)$, the time complexity is clearly $O\left(\left|A^{*}\right|\right)$ time. The size of every complete $D^{*}$-intersection is equal to $M$ from (44). From this discussion, the total time required for solving $\operatorname{WMI}\left(D^{*}\right)$ is $O\left(M\left|A^{*}\right|^{6}\right)$ from Theorem 2.3 .

\subsection{Results from [8]}

In this section, we introduce results concerning packing of in-trees given by Kamiyama et al. [8] which plays a crucial role in this paper.

Theorem $2.4([8])$ Given a directed graph $D=(V, A, S, f)$, the following three statements are equivalent:

1. For every $v \in V, \lambda\left(v, s^{*} ; D^{*}\right) \geq f\left(R_{D}(v)\right)$ holds. 
2. There exists a D-canonical set of arc-disjoint in-trees.

3. There exists a complete $D^{*}$-intersection.

Although the following theorem is not explicitly proved in [8], we can easily obtain it from the proof of Theorem 2.4 in [8].

Theorem 2.5 ([8]) Given a directed graph $D=(V, A, S, f)$ which satisfies the condition of Theorem 2.4, we can find a D-canonical set of arc-disjoint in-trees in $O\left(M^{2}|A|^{2}\right)$ time where $M=\sum_{v \in V} f\left(R_{D}(v)\right)$.

From Theorem 2.4, we obtain the following corollary.

Corollary 2.6 Given a directed graph $D=(V, A, S, f)$ and an arc set $B$ with $A \cap B=\emptyset$ which is parallel to $A$, the following three statements are equivalent:

1. B is a $D^{*}$-rooted connector.

2. There exists a $(D+B)$-canonical set of arc-disjoint in-trees.

3. There exists a complete $(D+B)^{*}$-intersection.

Proof. The equivalence of the statements 2 and 3 follows from Theorem 2.4.

$\mathbf{1} \rightarrow \mathbf{2}$ : Since $B$ is parallel to $A$, we clearly have

$$
(D+B)^{*}=D^{*}+B \text {. }
$$

Since $B$ is a $D^{*}$-rooted connector, we have for every $v \in V$

$$
\lambda\left(v, s^{*} ;(D+B)^{*}\right) \underbrace{=\lambda\left(v, s^{*} ; D^{*}+B\right)}_{\text {by (5) }} \geq f\left(R_{D}(v)\right) \underbrace{=f\left(R_{D+B}(v)\right)}_{\text {by (1) }} .
$$

From this inequality and Theorem 2.4, this part follows.

$\mathbf{2} \rightarrow \mathbf{1}$ : Since there exists a $(D+B)$-canonical set of arc-disjoint in-trees, we have for every $v \in V$

$$
\lambda\left(v, s^{*} ; D^{*}+B\right) \underbrace{=\lambda\left(v, s^{*} ;(D+B)^{*}\right)}_{\text {by (15) }} \underbrace{\geq f\left(R_{D+B}(v)\right)}_{\text {by Theorem [2.4 }} \underbrace{=f\left(R_{D}(v)\right)}_{\text {by (1) }} .
$$

This proves that $B$ is a $D^{*}$-rooted connector.

\section{An Algorithm for Covering by In-trees}

Given a directed graph $D=(V, A, S, f)$, we present in this section an algorithm for CDGI $(D)$. The time complexity of the proposed algorithm is bounded by a polynomial in $f(S)$ and the size of $D$. We first prove that $\operatorname{CDGI}(D)$ can be reduced to RAA-RA $\left(D^{*}\right)$. After this, we show that RAA-RA $\left(D^{*}\right)$ can be solved by using an algorithm for the weighted matroid intersection problem. 


\subsection{Reduction from CDGI to RAA-RA}

If $D=(V, A, S, f)$ is not $(S, f)$-proper, i.e., $\left|\delta_{D^{*}}(v)\right|>f\left(R_{D}(v)\right)$ for some $v \in V$, there exists no feasible solution of CDGI $(D)$ since there can not be a $D$-canonical set of in-trees that covers $\delta_{D^{*}}(v)$ from the definition of a $D$-canonical set of in-trees. Thus, we assume in the subsequent discussion that $D$ is $(S, f)$-proper.

Proposition 3.1 Given an $(S, f)$-proper directed graph $D=(V, A, S, f)$, the size of a $D^{*}$-rooted connector is at least $\sum_{v \in V} f\left(R_{D}(v)\right)-(|A|+f(S))$.

Proof. Let $B$ be a $D^{*}$-rooted connector. For every $v \in V,\left|\delta_{D^{*}+B}(v)\right| \geq f\left(R_{D}(v)\right)$ holds from the definition of a $D^{*}$-rooted connector. Thus, the number of $\operatorname{arcs}$ of $D^{*}+B$ is at least $\sum_{v \in V} f\left(R_{D}(v)\right)$. Since the number of $\operatorname{arcs}$ of $D^{*}$ is equal to $|A|+f(S)$ from (3) , the proposition holds.

For an $(S, f)$-proper directed graph $D=(V, A, S, f)$, we define opt ${ }_{D}$ by

$$
\mathrm{opt}_{D}=\sum_{v \in V} f\left(R_{D}(v)\right)-(|A|+f(S)) .
$$

From Proposition 3.1, the size of a $D^{*}$-rooted connector is at least opt $_{D}$.

Lemma 3.2 Given an $(S, f)$-proper directed graph $D=(V, A, S, f)$, there exists a feasible solution of $C D G I(D)$ if and only if there exists a $D^{*}$-rooted connector whose size is equal to opt ${ }_{D}$.

Proof. Only if-part: Suppose there exists a feasible solution of $\operatorname{CDGI}(D)$, i.e., there exists a $D$-canonical set $\mathcal{T}$ of in-trees which covers $A$. For each $i=1, \ldots, d$, we denote $f\left(s_{i}\right)\left(D, s_{i}\right)$-intrees of $\mathcal{T}$ by $T_{i, 1}, \ldots, T_{i, f\left(s_{i}\right)}$. For each $e \in A$, let $P_{e}=\left\{(i, j): e\right.$ is contained in $\left.T_{i, j}\right\}$. Since $\mathcal{T}$ covers $A$, each $e \in A$ is contained in at least one in-tree of $\mathcal{T}$. Thus, $\left|P_{e}\right| \geq 1$ holds for every $e \in A$. We define an arc set $B$ by $B=\bigcup_{e \in A}\left\{\left|P_{e}\right|-1\right.$ copies of $\left.e\right\}$. We will prove that $B$ is a $D^{*}$-rooted connector whose size is equal to opt ${ }_{D}$.

We first prove $|B|=\mathrm{opt}_{D}$. For this, we show that for every $v \in V$

$$
\sum_{e \in \delta_{D}(v)}\left(\left|P_{e}\right|-1\right)=f\left(R_{D}(v)\right)-\left|\delta_{D^{*}}(v)\right| .
$$

Let us first consider $v \notin S$. For $s_{i} \in R_{D}(v), T_{i, j}$ contains $v$ since $T_{i, j}$ spans $V_{D}^{i}$ and $s_{i}$ is reachable from $v$. Hence, since $T_{i, j}$ is an in-tree and $v$ is not a root of $T_{i, j}$ from $v \notin S, T_{i, j}$ contains exactly one arc $e \in \delta_{D}(v)$, i.e., $(i, j)$ is contained in $P_{e}$ for exactly one arc $e \in \delta_{D}(v)$. Thus, $\sum_{e \in \delta_{D}(v)}\left|P_{e}\right|=\sum_{s_{i} \in R_{D}(v)} f\left(s_{i}\right)=f\left(R_{D}(v)\right)$. From this equation and since $\left|\delta_{D}(v)\right|=\left|\delta_{D^{*}}(v)\right|$ follows from $v \notin S$, (17) holds. In the case of $v \in S$, for $s_{i} \in R_{D}(v) \backslash\{v\},(i, j)$ is contained in $P_{e}$ for exactly one $\operatorname{arc} e \in \delta_{D}(v)$ as in the case of $v \notin S$. Thus, $\sum_{e \in \delta_{D}(v)}\left|P_{e}\right|=f\left(R_{D}(v)\right)-f(v)$. From this equation and $\left|\delta_{D^{*}}(v)\right|=\left|\delta_{D}(v)\right|+f(v)$,

$$
\sum_{e \in \delta_{D}(v)}\left(\left|P_{e}\right|-1\right)=f\left(R_{D}(v)\right)-f(v)-\left|\delta_{D}(v)\right|=f\left(R_{D}(v)\right)-\left|\delta_{D^{*}}(v)\right| .
$$

This completes the proof of (17). Since $B$ contains $\left|P_{e}\right|-1$ copies of $e \in A$,

$$
\begin{aligned}
|B| & =\sum_{v \in V} \sum_{e \in \delta_{D}(v)}\left(\left|P_{e}\right|-1\right) \\
& =\sum_{v \in V}\left(f\left(R_{D}(v)\right)-\left|\delta_{D^{*}}(v)\right|\right) \quad(\text { from (17) }) \\
& =\operatorname{opt}_{D} \quad(\text { from (마) and (6) }) .
\end{aligned}
$$

What remains is to prove that $B$ is a $D^{*}$-rooted connector. From Corollary 2.6, it is sufficient to prove that there exists a $(D+B)$-canonical set of arc-disjoint in-trees. For this, we will 
construct from $\mathcal{T}$ a set $\mathcal{T}^{\prime}$ of arc-disjoint in-trees which consists of $T_{i, 1}^{\prime}, \ldots, T_{i, f\left(s_{i}\right)}^{\prime}$ for $i=1, \ldots, d$, and we prove that $\mathcal{T}^{\prime}$ is a $(D+B)$-canonical set of in-trees. Each $T_{i, j}^{\prime}$ is constructed from $T_{i, j}$ as follows. When $e \in A$ is contained in more than one in-tree of $\mathcal{T}$, in order to construct $\mathcal{T}^{\prime}$ from $\mathcal{T}$, we need to replace $e$ of $T_{i, j}$ by an arc in $B$ which is parallel to $e$ for every $(i, j) \in P_{e}$ except one in-tree. For $\left(i_{\min }, j_{\min }\right) \in P_{e}$ which is lexicographically smallest in $P_{e}$, we allow $T_{i_{\min }, j_{\min }}^{\prime}$ to use $e$, while for $(i, j) \in P_{e} \backslash\left(i_{\min }, j_{\min }\right)$, we replace $e$ of $T_{i, j}$ by an arc in $B$ which is parallel to $e$ so that for distinct $(i, j),\left(i^{\prime}, j^{\prime}\right) \in P_{e} \backslash\left(i_{\min }, j_{\min }\right)$, the resulting $T_{i, j}^{\prime}$ and $T_{i^{\prime}, j^{\prime}}^{\prime}$ contain distinct arcs which are parallel to $e$, respectively (see Figure 4).

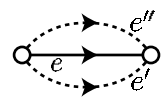

Figure 4: Illustration of the replacing operation. Let $e$ be an $\operatorname{arc}$ in $A$, and let $e^{\prime}, e^{\prime \prime}$ be $\operatorname{arcs}$ in $B$. Assume that $P_{e}=\{(1,1),(1,2),(2,1)\}$. In this case, $T_{1,1}, T_{1,2}$ and $T_{2,1}$ contain $e$. Then, $T_{1,1}^{\prime}$ contains $e$, $T_{1,2}^{\prime}$ contains $e^{\prime}$, and $T_{2,1}^{\prime}$ contains $e^{\prime \prime}$.

We will do this operation for every $e \in A$. Let $\mathcal{T}^{\prime}$ be the set of in-trees obtained by performing the above operation for every $e \in A$. Here we show that $\mathcal{T}^{\prime}$ is a $(D+B)$-canonical set of arc-disjoint in-trees. Since $T_{i, j}^{\prime}$ and $T_{i^{\prime}, j^{\prime}}^{\prime}$ are arc-disjoint for $(i, j) \neq\left(i^{\prime}, j^{\prime}\right)$ from the way of constructing $\mathcal{T}^{\prime}$, it is sufficient to prove that $T_{i, j}^{\prime}$ is a $\left(D+B, s_{i}\right)$-in-tree. Since $T_{i, j}^{\prime}$ is constructed by replacing arcs of $T_{i, j}$ by the corresponding parallel arc in $B$ and $T_{i, j}$ is an in-tree rooted at $s_{i}, T_{i, j}^{\prime}$ is also an in-tree rooted at $s_{i}$. Since $T_{i, j}$ spans $V_{D}^{i}$ and from (2), $T_{i, j}^{\prime}$ spans $V_{D+B}^{i}$. Hence, $T_{i, j}^{\prime}$ is a $\left(D+B, s_{i}\right)$-in-tree. This completes the proof.

If-part: Let $B$ be a $D^{*}$-rooted connector with $|B|=\mathrm{opt}_{D}$. From Corollary 2.6, there exists a $(D+B)$-canonical set $\mathcal{T}^{\prime}$ of arc-disjoint in-trees. For each $i=1, \ldots, d$, we denote $f\left(s_{i}\right)$ $\left(D+B, s_{i}\right)$-in-trees of $\mathcal{T}^{\prime}$ by $T_{i, 1}^{\prime}, \ldots, T_{i, f\left(s_{i}\right)}^{\prime}$. We will prove that we can construct from $\mathcal{T}^{\prime}$ a $D$-canonical set of in-trees covering $A$. We first construct from $\mathcal{T}^{\prime}$ a set $\mathcal{T}$ of in-trees which consists of $T_{i, j}$ for $i=1, \ldots, d$ and $j=1, \ldots, f\left(s_{i}\right)$ by the following procedure Replace.

Procedure Replace: For each $i=1, \ldots, d$ and $j=1, \ldots, f\left(s_{i}\right)$, set $T_{i, j}$ to be a directed graph obtained from $T_{i, j}^{\prime}$ by replacing every arc $e \in B$ which is contained in $T_{i, j}^{\prime}$ by an arc in $A$ which is parallel to $e$.

From now on, we prove that $\mathcal{T}$ is a $D$-canonical set of in-trees which covers $A$. It is not difficult to prove that $\mathcal{T}$ is a $D$-canonical set of in-trees from the definition of the procedure Replace in the same manner as the last part of the proof of the "only if-part". Thus, it is sufficient to prove that $\mathcal{T}$ covers $A$. For this, we first show that $\mathcal{T}^{\prime}$ covers $A \cup B$. From $A \cap B=\emptyset,|B|=\mathrm{opt}_{D}$ and (6),

$$
|A \cup B|=|A|+\mathrm{opt}_{D}=\sum_{v \in V} f\left(R_{D}(v)\right)-f(S) .
$$

Recall that each $v \in V$ is contained in $f\left(R_{D+B}(v)\right)$ in-trees of $\mathcal{T}^{\prime}$ from the definition of a $(D+B)$-canonical set of in-trees. Thus, since in-trees of $\mathcal{T}^{\prime}$ are arc-disjoint, it holds for each $v \in V$ that the number of $\operatorname{arcs}$ in $\delta_{D+B}(v)$ which are contained in in-trees of $\mathcal{T}^{\prime}$ is equal to

$$
\begin{cases}f\left(R_{D+B}(v)\right), & \text { if } v \in V \backslash S \\ f\left(R_{D+B}(v)\right)-f(v), & \text { if } v \in S\end{cases}
$$


Hence, the number of $\operatorname{arcs}$ in $A \cup B$ contained in in-trees of $\mathcal{T}^{\prime}$ is equal to

$$
\begin{aligned}
& \sum_{v \in V \backslash S} f\left(R_{D+B}(v)\right)+\sum_{v \in S}\left(f\left(R_{D+B}(v)\right)-f(v)\right) \\
& \left.=\sum_{v \in V} f\left(R_{D+B}(v)\right)-f(S)=\sum_{v \in V} f\left(R_{D}(v)\right)-f(S) \quad \text { (from (1) }\right) .
\end{aligned}
$$

Since any arc of $\mathcal{T}^{\prime}$ is in $A \cup B$ and the number of arcs in $A \cup B$ is equal to that of $\mathcal{T}^{\prime}$ from (8) and (10), $\mathcal{T}^{\prime}$ contains all arcs in $A$. Thus, $\mathcal{T}$ covers $A$ from the definition of the procedure Replace.

As seen in the proof of the "if-part" of Lemma 3.2, if we can find a $D^{*}$-rooted connector $B$ with $|B|=\mathrm{opt}_{D}$, we can compute a $D$-canonical set of in-trees which covers $A$ by using the procedure Replace from a $(D+B)$-canonical set of arc-disjoint in-trees. Furthermore, we can construct a $(D+B)$-canonical set of arc-disjoint in-trees by using the algorithm of Theorem 2.5 . Since the optimal value of RAA-RA $\left(D^{*}\right)$ is at least opt ${ }_{D}$ from Proposition 3.1, we can test if there exists a $D^{*}$-rooted connector whose size is equal to opt ${ }_{D}$ by solving RAA-RA $\left(D^{*}\right)$. Assuming that we can solve RAA-RA $\left(D^{*}\right)$, our algorithm for finding a $D$-canonical set of in-trees which covers $A$ called Algorithm CR can be illustrated as Algorithm 1 below.

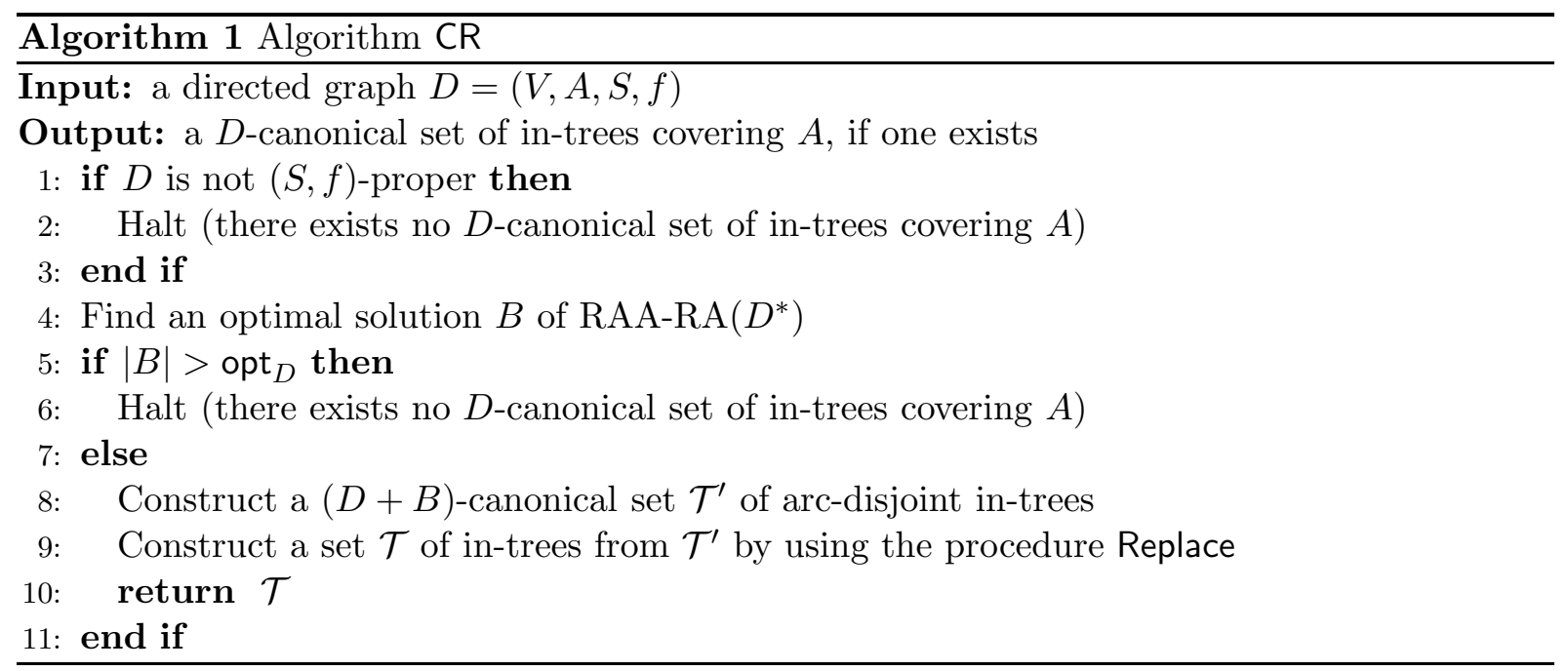

Lemma 3.3 Given a directed graph $D=(V, A, f, S)$, Algorithm CR correctly finds a D-canonical set of in-trees which covers $A$ in $O\left(\gamma_{1}+|V||A|+M^{4}\right)$ time if one exists where $\gamma_{1}$ is the time required to solve $R A A-R A\left(D^{*}\right)$ and $M=\sum_{v \in V} f(R(v))$.

Proof. The correctness of the algorithm follows from Lemma 3.2, Thus, we consider the time complexity. In Step 1, we have to compute $R_{D}(v)$ for every $v \in V$. This can be done in $O(|V||A|)$ time by applying depth-first search from every $s_{i} \in S$. After this, the time required to test whether $\left|\delta_{D^{*}}(v)\right| \leq f\left(R_{D}(v)\right)$ for all $v \in V$ is $O(|A|)$. Thus, the time required for Step 1 is $O(|V||A|)$. Since the number of $\operatorname{arcs}$ of $D+B$ is at most $M$ for a $D^{*}$-rooted connector $B$ with $|B|=\mathrm{opt}_{D}$ from (6), the time required for Step 8 is $O\left(M^{4}\right)$ from Theorem 2.5. Moreover, since the number of arcs of $D+B$ is at most $M$, the time required for Step 9 is $O(M)$ from the definition of Procedure Replace. Hence, since the time required for Step 4 is $\gamma_{1}$, the lemma follows. 


\subsection{Reduction from RAA-RA to WMI}

From the algorithm CR in Section 3.1, in order to present an algorithm for $\operatorname{CDGI}(D)$, what remains is to show how we solve RAA-RA $\left(D^{*}\right)$. In this section, we will prove that we can test whether there exists a $D^{*}$-rooted connector whose size is equal to opt ${ }_{D}$ (i.e., Steps 4 and 5 in the algorithm CR) by reducing it to the problem WMI. Our proof is based on the algorithm of [3] for RAA-RA $\left(D^{*}\right)$ in which $S$ consists of a single vertex. We extend the idea of [3] to the case of $|S|>1$ by using Theorem 2.4. We define a directed graph $D_{+}$obtained from $D$ by adding opt $D$ parallel arcs to every $e \in A$. Then, we will compute a $D^{*}$-rooted connector whose size is equal to opt ${ }_{D}$ by using an algorithm for $\mathrm{WMI}\left(D_{+}^{*}\right)$ as described below. Since the number of arcs in a $D^{*}$-rooted connector whose size is equal to $\mathrm{opt}_{D}$ which are parallel to one arc in $A$ is at most opt $_{D}$, it is enough to add opt $D$ parallel arcs to each arc of $A$ in $D_{+}$in order to find a $D^{*}$-rooted connector whose size is equal to opt ${ }_{D}$.

We denote by $A_{+}$and $A_{+}^{*}$ the arc sets of $D_{+}$and $D_{+}^{*}$, respectively. If $I \subseteq A_{+}^{*}$ is a complete $D_{+}^{*}$-intersection, since $I$ is a base of $\boldsymbol{U}\left(D_{+}^{*}\right)$ and from (4) and (1),

$$
|I|=\sum_{v \in V} f\left(R_{D_{+}}(v)\right)=\sum_{v \in V} f\left(R_{D}(v)\right) .
$$

We define a weight function $w: A_{+}^{*} \rightarrow \mathbb{R}_{+}$by

$$
w(e)= \begin{cases}0, & \text { if } e \in A^{*} \\ 1, & \text { otherwise }\end{cases}
$$

The following lemma shows the relation between $\operatorname{RAA}-\mathrm{RA}\left(D^{*}\right)$ and $\operatorname{WMI}\left(D_{+}^{*}\right)$.

Lemma 3.4 Given an $(S, f)$-proper directed graph $D=(V, A, S, f)$, there exists a $D^{*}$-rooted connector whose size is equal to $\mathrm{opt}_{D}$ if and only if there exists a complete $D_{+}^{*}$-intersection whose weight is equal to opt ${ }_{D}$.

To prove Lemma 3.4, we need to show the following two lemmas.

Lemma 3.5 Given a directed graph $D=(V, A, S, f)$ and an arc set $B$ which is parallel to $A$,

1. if there is a complete $D^{*}$-intersection $I, I$ is also a complete $(D+B)^{*}$-intersection, and

2. if there is a complete $(D+B)^{*}$-intersection $I$ such that $I \subseteq A^{*}, I$ is also a complete $D^{*}$-intersection.

Proof. 1: We first prove that $I$ is a base of $\boldsymbol{M}\left((D+B)^{*}\right)$. Since $I$ is a base of $\boldsymbol{M}\left(D^{*}\right), I$ can be partitioned into $\left\{I_{i, 1}, \ldots, I_{i, f\left(s_{i}\right)}: i=1, \ldots, d\right\}$ such that a directed graph $\left(V_{D}^{i} \cup\left\{s^{*}\right\}, I_{i, j}\right)$ is a tree for every $i=1, \ldots, d$ and $j=1, \ldots, f\left(s_{i}\right)$. Thus, since each $\left(V_{D+B}^{i} \cup\left\{s^{*}\right\}, I_{i, j}\right)$ is a tree from (2), $I$ is a base of $\boldsymbol{M}\left((D+B)^{*}\right)$.

Next we prove that $I$ is a base of $\boldsymbol{U}\left((D+B)^{*}\right)$. Since $I$ is a base of $\boldsymbol{U}\left(D^{*}\right),\left|\delta_{D^{*}}(v) \cap I\right|$ is equal to

$$
\begin{cases}f\left(R_{D}(v)\right), & \text { if } v \in V \\ 0, & \text { if } v=s^{*}\end{cases}
$$

Furthermore, since $I \cap B=\emptyset$ follows from $I \subseteq A^{*},\left|\delta_{D^{*}}(v) \cap I\right|$ is equal to $\left|\delta_{(D+B)^{*}}(v) \cap I\right|$ for every $v \in V$. Thus, for each $v \in V,\left|\delta_{(D+B)^{*}}(v) \cap I\right|$ is equal to

$$
\begin{cases}f\left(R_{D}(v)\right)=f\left(R_{D+B}(v)\right), & \text { if } v \in V \\ 0, & \text { if } v=s^{*}\end{cases}
$$


This proves that $I$ is a base of $\boldsymbol{U}\left((D+B)^{*}\right)$.

2: This part can be proved in the same manner as in the proof of the part 1 .

巳 巳

Lemma 3.6 Given $D_{+}^{*}$ of an $(S, f)$-proper directed graph $D=(V, A, S, f)$ and a weight function $w: A_{+}^{*} \rightarrow \mathbb{R}_{+}$defined by (12), if there exists a complete $D_{+}^{*}$-intersection $I \subseteq A_{+}^{*}, w(I) \geq \mathrm{opt}_{D}$. Moreover, $w(I)=\mathrm{opt}_{D}$ if and only if $A^{*} \subseteq I$.

Proof. From (12), we have $w(I)=|I|-\left|I \cap A^{*}\right|$. Furthermore,

$$
|I|-\left|I \cap A^{*}\right| \geq|I|-\left|A^{*}\right| \underbrace{=\sum_{v \in V} f\left(R_{D}(v)\right)-(|A|+f(S))}_{\text {from (3) } \text { and (11) }} .
$$

Thus, $w(I) \geq \mathrm{opt}_{D}$ follows from (6). From the above equation, $w(I)=\mathrm{opt}_{D}$ if and only if $\left|I \cap A^{*}\right|=\left|A^{*}\right|$. This proves the rest of the lemma.

Proof of Lemma 3.4. Only if-part: Assume that there exists a $D^{*}$-rooted connector whose size is equal to $\mathrm{opt}_{D}$. Since $D_{+}$has opt $D$ parallel arcs to every $e \in A$, there exists a $D^{*}$-rooted connector $B \subseteq A_{+} \backslash A$ with $|B|=$ opt $_{D}$. Let us fix a $D^{*}$-rooted connector $B \subseteq A_{+} \backslash A$ with $|B|=\mathrm{opt}_{D}$. From (i) of Lemma 3.5, in order to prove the "only if-part", it is sufficient to prove that there exists a complete $(D+B)^{*}$-intersection $I$ with $w(I)=\mathrm{opt}_{D}$. Since there exists a complete $(D+B)^{*}$-intersection $I$ from Corollary 2.6, we will prove that $w(I)=\mathrm{opt}_{D}$. Since the arc set of $(D+B)^{*}$ is equal to $A^{*} \cup B$ and $I$ is a $(D+B)^{*}$-intersection, $I \subseteq A^{*} \cup B$ holds. Thus, since $w\left(A^{*} \cup B\right)=|B|=\mathrm{opt}_{D}$ follows from (12), $w(I) \leq w\left(A^{*} \cup B\right)=\mathrm{opt}_{D}$ holds. Hence, $w(I)=\mathrm{opt}_{D}$ follows from Lemma 3.6. This completes the proof.

If-part: Assume that there exists a complete $D_{+}^{*}$-intersection $I$ with $w(I)=\operatorname{opt}_{D}$. Let $B$ be $I \backslash A^{*}$, and we will prove that $B$ is a $D^{*}$-rooted connector with $|B|=\mathrm{opt}_{D}$. We first prove $B$ is a $D^{*}$-rooted connector by using (ii) of Lemma 3.5] and Corollary 2.6. We set $B$ and $D$ in Lemma 3.5 to be $A_{+} \backslash(A \cup B)$ and $D+B$, respectively. Notice that $(D+B)+\left(A_{+} \backslash(A \cup B)\right)=D_{+}$ follows from $B \subseteq A_{+}$and $A_{+} \backslash(A \cup B)$ is parallel to $A \cup B$. From $B=I \backslash A^{*}$, we have $I \subseteq A^{*} \cup B$. Thus, $I$ is a complete $(D+B)^{*}$-intersection since $I$ is a complete $D_{+}^{*}$-intersection and from (ii) of Lemma 3.5. Hence, from Corollary 2.6, $B$ is a $D^{*}$-rooted connector.

What remains is to prove that $|B|=\mathrm{opt}_{D}$. From Lemma 3.6 and $w(I)=\mathrm{opt}_{D}, A^{*} \subseteq I$ holds. Thus, from $B=I \backslash A^{*}$ and (11),

$$
|B|=\left|I \backslash A^{*}\right|=|I|-\left|A^{*}\right|=\sum_{v \in V} f\left(R_{D}(v)\right)-(|A|+f(S)) .
$$

This equation and (6) complete the proof.

As seen in the proof of the "if-part" of Lemma 3.4, if we can find a complete $D_{+}^{*}$-intersection $I$ with $w(I)=$ opt $_{D}$, we can find a $D^{*}$-rooted connector $B$ with $|B|=$ opt $_{D}$ by setting $B=I \backslash A^{*}$. Furthermore, we can obtain a complete $D_{+}^{*}$-intersection whose weight is equal to opt ${ }_{D}$ if one exists by using the algorithm for $\mathrm{WMI}\left(D_{+}^{*}\right)$ since the optimal value of $\mathrm{WMI}\left(D_{+}^{*}\right)$ is at least opt ${ }_{D}$ from Lemma 3.6. The formal description of the algorithm called Algorithm RW for finding a $D^{*}$-rooted connector whose size is equal to opt ${ }_{D}$ is illustrated in Algorithm 2 .

Lemma 3.7 Given $D^{*}$ of an $(S, f)$-proper directed graph $D=(V, A, f, S)$, Algorithm RW correctly finds a $D^{*}$-rooted connector whose size is equal to opt ${ }_{D}$ in $O\left(\gamma_{2}+M|A|\right)$ time if one exists where $\gamma_{2}$ is the time required to solve $W M I\left(D_{+}^{*}\right)$ and $M=\sum_{v \in V} f\left(R_{D}(v)\right)$. 


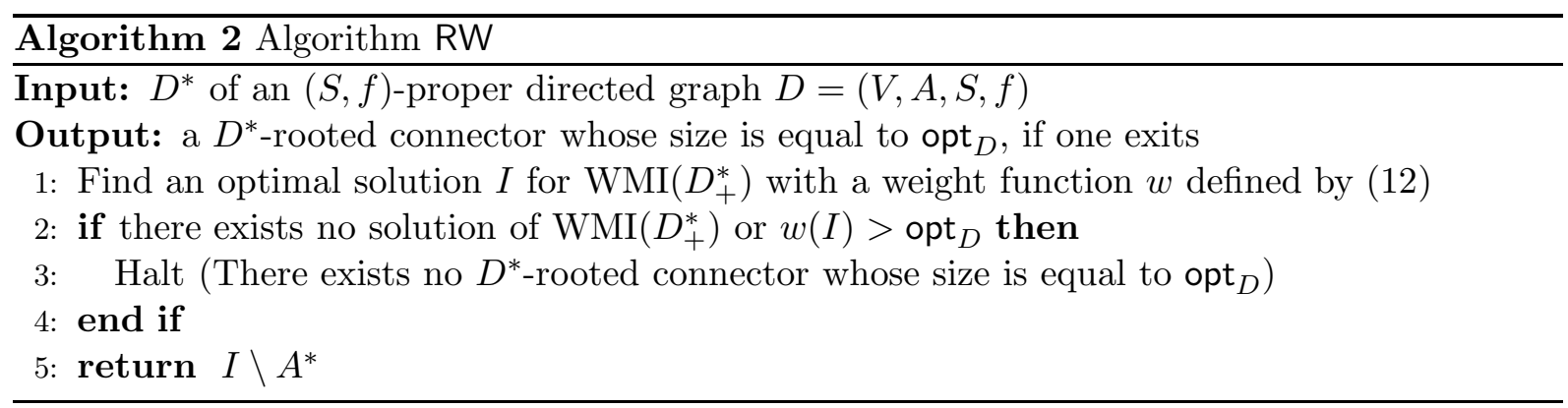

Proof. The correctness of the algorithm follows from Lemma 3.4. We consider the time complexity. In Step 1, we can construct $D_{+}^{*}$ in $O(M|A|)$ time since $D_{+}^{*}$ has opt ${ }_{D}$ arcs parallel to each arc in $A$ and from (6). Hence, since the time required for Step 2 is equal to $\gamma_{2}$, the lemma holds.

\subsection{Algorithm for CDGI}

We are ready to explain the formal description of our algorithm called Algorithm Covering for $\operatorname{CDGI}(D)$. Algorithm Covering is the same as Algorithm CR such that Steps 4, 5 and 6 are replaced by Algorithm RW.

Theorem 3.8 Given a directed graph $D=(V, A, S, f)$, Algorithm Covering correctly finds a D-canonical set of in-trees which covers $A$ in $O\left(M^{7}|A|^{6}\right)$ time if one exits where $M=$ $\sum_{v \in V} f\left(R_{D}(v)\right)$.

Proof. The correctness of the algorithm follows from Lemmas 3.3 and 3.7. We then consider the time complexity of this algorithm. From Lemmas 3.3 and 3.7, what remains is to analyze the time required to solve $\operatorname{WMI}\left(D_{+}^{*}\right)$. If $D$ is $(S, f)$-proper, $\left|A^{*}\right|=\sum_{v \in V}\left|\delta_{D^{*}}(v)\right| \leq \sum_{v \in V} f\left(R_{D}(v)\right)=M$. Thus, since $D_{+}^{*}$ has opt $D$ parallel arcs of every $e \in A,\left|A_{+}^{*}\right|=\left|A^{*}\right|+\sum_{e \in A^{\circ}} \mathrm{opt}_{D} \leq M+M|A|$. Hence we have $\left|A_{+}^{*}\right|=O(M|A|)$. Thus, from Lemma 2.1, we can solve $\operatorname{WMI}\left(D^{*}\right)$ in $O\left(M^{7}|A|^{6}\right)$ time. From this discussion and Lemmas 3.3 and 3.7, we obtain the theorem.

\section{Acyclic Case}

In this section, we show that in the case where $D=(V, A, S, f)$ is acyclic, a $D$-canonical set of in-trees covering $A$ can be computed more efficiently than the general case. For this, we prove the following theorem.

Theorem 4.1 Given an acyclic directed graph $D=(V, A, S, f)$, there exists a $D$-canonical set of in-trees which covers $A$ if and only if

$$
|B| \leq f\left(R_{D}\left(\partial^{+}(B)\right)\right) \text { for every } v \in V \text { and } B \subseteq \delta_{D}(v) \text {. }
$$

Proof. For each $v \in V$, we define an undirected bipartite graph $G_{v}=\left(X_{v} \cup Y_{v}, E_{v}\right)$ which is necessary to prove the theorem. Let $X_{v}=\left\{x_{e}: e \in \delta_{D}(v)\right\}$ and $Y_{v}=\left\{y_{i, j}: s_{i} \in R_{D}(v), j=\right.$ $\left.1, \ldots, f\left(s_{i}\right)\right\} . x_{e} \in X_{v}$ and $y_{i, j} \in Y_{v}$ are connected by an edge in $E_{v}$ if and only if $s_{i}$ is reachable from $\partial^{+}(e)$ (see Figure 5 ). 


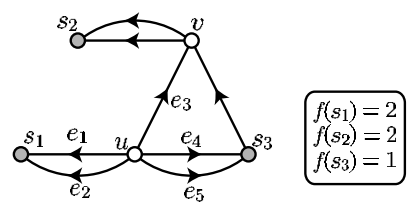

(a)

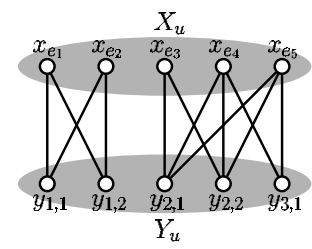

(b)

Figure 5: (a) Input acyclic directed graph D. (b) Bipartite graph $G_{u}$ for $u$ in (a).

It is well-known that (14) is equivalent to the necessary and sufficient condition that for any $v \in V$, there exists a matching in $G_{v}$ which saturates vertices in $X_{v}$ (e.g., Theorem 16.7 in Chapter 16 of [12]). Thus it is sufficient to prove that there exists a $D$-canonical set of in-trees which covers $A$ if and only if for any $v \in V$, there exists a matching in $G_{v}$ which saturates vertices in $X_{v}$.

If-part: Since $D$ has no cycle, we can label vertices in $V$ as follows, based on topological ordering: (i) A label of each vertex is an integer between 1 and $|V|$. (ii) For any $e \in A$, a label of $\partial^{+}(e)$ is smaller than that of $\partial^{-}(e)$. For $W \subseteq V$, we denote by $D[W]$ a subgraph of $D=(V, A, S, f)$ induced by $W$ with a set of specified vertices $S \cap W$ and a restriction of $f$ on $S \cap W$. Let $V_{t}$ be the set of all vertices whose label is at most $t$. We prove by induction on $t$. For $t=1$, it is clear that there exists a $D\left[V_{1}\right]$-canonical set of in-trees covering the arc set of $D\left[V_{1}\right]$. Assume that in the case of $t \geq 1$, there exists a $D\left[V_{t}\right]$-canonical set $\mathcal{T}$ of in-trees covering the arc set of $D\left[V_{t}\right]$. For $s_{i} \in S \cap V_{t}$ and $j=1, \ldots, f\left(s_{i}\right)$, let $T_{i, j}$ be an in-tree of $\mathcal{T}$ which is rooted at $s_{i}$ and spans vertices in $V_{t}$ from which $s_{i}$ is reachable.

Let $v$ be a vertex whose label is equal to $t+1$.

Case1: We first consider the case of $v \notin S$. In this case, from $S \cap V_{t}=S \cap V_{t+1}$, we will construct a set $\mathcal{T}^{\prime}$ of in-trees which consists of $T_{i, 1}^{\prime}, \ldots, T_{i, f\left(s_{i}\right)}^{\prime}$ for $s_{i} \in S \cap V_{t}\left(=S \cap V_{t+1}\right)$ such that each $T_{i, j}^{\prime}$ is obtained from $T_{i, j}$. We first consider $T_{i, j}^{\prime}$ for $s_{i} \in\left(S \cap V_{t}\right) \backslash R_{D}(v)$. For $s_{i} \in\left(S \cap V_{t}\right) \backslash R_{D}(v)$, from $V_{D\left[V_{t}\right]}^{i}=V_{D\left[V_{t+1}\right]}^{i}$ holds, $T_{i, j}$ is also a $\left(D\left[V_{t+1}\right], s_{i}\right)$-in-tree. Thus, we set $T_{i, j}^{\prime}=T_{i, j}$. Next we consider $T_{i, j}^{\prime}$ for $s_{i} \in R_{D}(v)$. For $s_{i} \in R_{D}(v)$, since $V_{D\left[V_{t+1}\right]}^{i}=V_{D\left[V_{t}\right]}^{i} \cup\{v\}$ holds, we need to add an arc in $\delta_{D}(v)$ to $T_{i, j}$. Here we use a matching $\mathcal{M}$ in $G_{v}$ which saturates vertices in $X_{v}$. For each edge $x_{e} y_{i, j} \in \mathcal{M}$, we set $T_{i, j}^{\prime}$ be an in-tree obtained by adding an arc $e$ to $T_{i, j}$. If there exists $y_{i^{\prime}, j^{\prime}} \in Y_{v}$ which is not contained in any edge in $\mathcal{M}$, we arbitrarily choose an $\operatorname{arc} e^{\prime} \in \delta_{D}(v)$ such that $x_{e^{\prime}}$ is a neighbour of $y_{i^{\prime}, j^{\prime}}$ in $G_{v}$ and we set $T_{i^{\prime}, j^{\prime}}^{\prime}$ to be an in-tree obtained by adding $e^{\prime}$ to $T_{i^{\prime}, j^{\prime}}^{\prime}$. From the way of construction, $\mathcal{T}^{\prime}$ is clearly a $D\left[V_{t+1}\right]$-canonical set of in-trees. Since $M$ saturates vertices in $X_{v}, T_{i, 1}^{\prime}, \ldots, T_{i, f\left(s_{i}\right)}^{\prime}$ with $s_{i} \in R_{D}(v)$ contain all $\operatorname{arcs}$ in $\delta_{D}(v)$. Thus, since $\mathcal{T}$ covers the arc set of $D\left[V_{t}\right]$ from the induction hypothesis, $\mathcal{T}^{\prime}$ covers the arc set of $D\left[V_{t+1}\right]$.

Case2: Next we consider the case of $v \in S$. In this case, since $\left(S \cap V_{t}\right) \backslash\left(S \cap V_{t+1}\right)=\{v\}$ holds, letting $v=s_{i}$, we need to add new in-trees $T_{i, j}^{\prime}=\left(\left\{s_{i}\right\}, \emptyset\right)$ for every $j=1, \ldots, f\left(s_{i}\right)$ to $\mathcal{T}^{\prime}$ which is constructed as above. This completes the proof of the "if-part".

Only if-part: Assume that there exists a $D$-canonical set $\mathcal{T}$ of in-trees covering $A$. For $i=1, \ldots, d$, we denote $f\left(s_{i}\right)\left(D, s_{i}\right)$-in-trees of $\mathcal{T}$ by $T_{i, 1}, \ldots, T_{i, f\left(s_{i}\right)}$. Let us fix $v \in V$, and for $X_{v}$ and $Y_{v}$ we define a set $E^{\prime}$ in which an edge $x_{e} y_{i, j}$ is contained in $E^{\prime}$ if and only if $e \in \delta_{D}(v)$ is contained in $T_{i, j}$. If $e \in \delta_{D}(v)$ is contained in $T_{i, j}, s_{i}$ is reachable from $\partial^{+}(e)$. Thus, $E^{\prime}$ is a subset of $E_{v}$. Since $\mathcal{T}$ covers $A$, each $e \in \delta_{D}(v)$ is contained in at least one in-tree in $\mathcal{T}$. That is, $E^{\prime}$ saturates $X_{v}$. Since $T_{i, j}$ is an in-tree, each $y_{i, j}$ is contained in exactly one edge in $E^{\prime}$. Thus, it is not difficult to see that a matching in $G_{v}$ which saturates vertices in $X_{v}$ can be obtained 
from $E^{\prime}$. This completes the proof.

From Theorem 4.1, instead of the algorithm presented in Section 3, we can more efficiently find a $D$-canonical set of in-trees covering $A$ by finding a maximum matching in a bipartite graph $O(|V|)$ times. In regard to algorithms for finding a maximum matching in a bipartite graph, see e.g. [6].

Corollary 4.2 Given an acyclic directed graph $D=(V, A, S, f)$, we can find a $D$-canonical set of in-trees which covers $A$ in $O(\operatorname{match}(M+|A|, M|A|))$ time if one exists where match $(n, m)$ represents the time required to find maximum matching in a bipartite graph with $n$ vertices and $m$ arcs and $M=\sum_{v \in V} f\left(R_{D}(v)\right)$.

Proof. From the proof of Theorem 4.1, for each $v \in V,\left|X_{v}\right|=\left|\delta_{D}(v)\right|$ and $\left|Y_{v}\right|=f\left(R_{D}(v)\right)$ hold. Then, $\left|E_{v}\right|=O\left(\left|\delta_{D}(v)\right| \cdot f\left(R_{D}(v)\right)\right)$ follows. Thus, the corollary follows from $\sum_{v \in V}\left(\left|X_{v}\right|+\left|Y_{v}\right|\right)=$ $M+|A|$ and $\sum_{v \in V}\left|E_{v}\right|=M|A|$.

Acknowledgement: We thank Prof. Tibor Jordán who informed us of the paper [3] and we are grateful to Shin-ichi Tanigawa for helpful comments.

\section{References}

[1] E. M. Arkin, R. Hassin, and A. Levin. Approximations for minimum and min-max vehicle routing problems. J. Algorithms, 59(1):1-18, 2006.

[2] G. Even, N. Garg, J. Könemann, R. Ravi, and A. Sinha. Min-max tree covers of graphs. Oper. Res. Lett., 32(4):309-315, 2004.

[3] A. Frank. Rooted $k$-connections in digraphs. Discrete Applied Mathematics. (to appear).

[4] A. Frank. Covering branchings. Acta Scientiarum Mathematicarum [Szeged], 41:77-81, 1979.

[5] A. Frank. A weighted matroid intersection algorithm. J. Algorithms, 2(4):328-336, 1981.

[6] J. E. Hopcroft and R. M. Karp. An $n^{5 / 2}$ algorithm for maximum matchings in bipartite graphs. SIAM J. Comput., 2(4):225-231, 1973.

[7] T. Jordan. Two $\mathcal{N} \mathcal{P}$-complete augmentation problems. Technical Report 8, Department of Mathematics and Computer Science, Odense University, 1997.

[8] N. Kamiyama, N. Katoh, and A. Takizawa. Arc-disjoint in-trees in directed graphs. In Proc. the nineteenth Annual ACM-SIAM Symposium on Discrete Algorithms (SODA2008), pages 518-526, 2008.

[9] D.E. Knuth. Matroid partitioning. Technical Report STAN-CS-73-342, Computer Science Department, Stanford University, 1974.

[10] H. Nagamochi and K. Okada. Approximating the minmax rooted-tree cover in a tree. Inf. Process. Lett., 104(5):173-178, 2007.

[11] J. G. Oxley. Matroid theory. Oxford University Press, 1992. 
[12] A. Schrijver. Combinatorial Optimization: Polyhedra and Efficiency (Algorithms and Combinatorics). Springer-Verlag, 2003.

[13] K. Vidyasankar. Covering the edge set of a directed graph with trees. Discrete Mathematics, 24:79-85, 1978. 